INRA Prod. Anim., 1992, 5 (3), 173 - 178

\section{K. BENABDELJELIL, P. MÉRAT* Institut Agronomique et Vétérinaire Hassan II, \\ Département des Productions Animales, B.P. 6202 Rabat Instituts, Maroc}

*INRA Laboratoire de Génétique

Factorielle

78352 Jouy-en-Josas Cedex

\title{
Test de types génétiques pour une production avicole locale au Maroc
}

Des résultats sur les effets associés à des gènes spécifiques comme $\mathrm{Na}$ (Cou nu) et dw (nanisme lié au sexe) selon la température ambiante ont été publiés à diverses reprises (voir revues par Mérat 1986, Rauen et al 1986, Mérat 1990). Les recherches correspondantes nécessitaient des lignées expérimentales pour comparer les génotypes étudiés dans le même contexte génétique moyen, et faisaient appel à des environnements contrôlés tels que des chambres climatiques. Des résultats de nature différente et complémentaires des premiers peuvent faire appel à des croisements de type commercial contenant ou non les gènes potentiellement intéressants, et à un "testage" en conditions locales d'élevage et de climat. Ce second type d'investigations ne permet pas des interprétations aussi rigoureuses que le premier dans le domaine génétique, mais son choix peut être guidé par les résultats de recherche antérieurs, et son interprétation peut être ensuite suggérée par eux. C'est dans cet esprit que nous avons réalisé des tests comparatifs de deux types géné-

\section{Résumé}

Un croisement de poulets de chair de type Label "Cou nu" et un croisement de type commercial standard ont été testés tous deux dans des conditions d'élevage courantes au Maroc, d'une part en saison d'hiver (éclosion le 19/01/90), de l'autre en saison chaude (éclosion le $05 / 06 / 90$ ). La croissance, l'efficacité alimentaire et la mortalité ont été relevées jusqu'à l'âge de 70 jours dans le premier cas, de 49 jours dans le second. Les performances de croissance des deux croisements, comme prévisible, ont été très différentes. L'effet dépressif de la saison chaude sur le poids corporel et l'efficacité alimentaire a été moins marqué pour le type "Label", qui a présenté par ailleurs une très faible mortalité aux deux saisons.

D'autre part, un croisement expérimental de type "pondeuse à oeufs bruns" réunissant les gènes "nanisme lié au sexe" ( $\mathrm{dw}$ ) et "Cou nu" (Na) a été testé pour des performances de production d'oeufs dans des conditions d'élevage courantes au Maroc, en même temps qu'un croisement "ponte" commercial , également de type "oeuf brun" (ISA-Brown). La persistance de ponte et le poids moyen des oeufs du croisement expérimental ont été inférieurs à ceux du croisement commercial. Par contre, le poids corporel et la consommation alimentaire plus faibles du croisement expérimental ont contribué à réduire le désavantage de ce type génétique pour l'efficacité alimentaire. Le taux de ponte a été légèrement favorable au croisement "naines Cou nu" jusqu'à l'âge de 35 semaines, probablement du fait d'une bonne thermotolérance associée aux gènes $\mathrm{dw}$ et $\mathrm{Na}$. Les données suggèrent une moindre mortalité pour ce même croisement après l'âge de $\mathbf{4 0}$ semaines. tiques, l'un avec l'apport des gène "Cou nu" et/ou "nanisme" et l'autre non, pour l'élevage au Maroc, à deux saisons différentes, de poulets de chair. Une comparaison analogue a été réalisée sur d'autres types génétiques pour la production d'oeufs.

\section{1 / Poulets de chair}

\section{1 / Conditions expérimentales}

\section{a / Types génétiques comparés}

Les performances d'un croisement comportant le gène "Cou nu" ont été comparées à celles d'un croisement "chair" classique. Le premier croisement était de type "Label" (croissance plus lente que le type industriel, abattage plus tardif) toutefois plus lourd que les "Label" courants. Ce croisement (431 : lignée mâle T44 Cou nu, lignée maternelle alourdie) fourni par la SASSO, a été comparé au type industriel ISA 715 (issu d'une lignée mâle Cornish et de la poule nanifiée "Vedette").

\section{b / Conditions d'élevage}

Deux essais ont été réalisés : le premier en saison relativement froide (éclosion le $10 / 01 / 90$ ), le second en saison chaude (éclosion le 05/06/90). Dans les deux cas, les poussins éclos en France ont été envoyés par avion au Maroc.

L'élevage, de type industriel courant au Maroc, comportait deux bâtiments en dur avec fenêtres, faciles à ventiler mais non climatisés. Chacun était divisé en 9 loges permettant la mesure de consommations alimentaires en groupes. Les densités réalisées ont été volontairement inférieures dans le second essai (saison chaude) : 15 animaux $/ \mathrm{m}^{2}$ pour le croisement 431,13 pour le croisement 715 plus lourd dans le premier essai ; respectivement 12,5 et 11,5 pour les mêmes croisements dans la seconde série. L'élevage était fait au sol sur litière de paille. 
Un aliment "croissance", contenant approximativement $21 \%$ de matières azotées et $2850 \mathrm{kcal}$ d'énergie métabolisable $(\mathrm{EM}) / \mathrm{kg}$, était distribué ad libitum sous forme de farine dans les deux séries d'élevage jusqu'à 49 jours. Après cet âge, les poulets de la première série, qui ont pu être élevés jusqu'à 70 jours, recevaient un aliment "finition" à $17,9 \%$ de matières azotées et $2950 \mathrm{kcal}$ $\mathrm{EM} / \mathrm{kg}$. Un tiers des poulets (3 répétitions par type génétique) recevait cet aliment de base ; deux autres tiers recevaient ce même aliment supplémenté respectivement par $200 \mathrm{ppm}$ d'acide ascorbique de deux origines différentes.

Les poussins étaient éclairés 23 heures par jour à raison de $4 \mathrm{w} / \mathrm{m}^{2}$ en supplément du jour naturel. Ils étaient vaccinés contre la maladie de NewCastle et la bronchite infectieuse.

Dans le premier essai, les minima journaliers de température descendaient jusqu'à $12^{\circ} \mathrm{C}$ et les maxima journaliers n'excédaient pas $26^{\circ} \mathrm{C}$ ( $30^{\circ} \mathrm{C}$ une journée). Dans le second essai, les minima journaliers ont été de $22^{\circ} \mathrm{C}$ et les maxima ont atteint $42^{\circ} \mathrm{C}$, les moyennes journalières fluctuant entre $25^{\circ}$ et $34^{\circ}$. Le degré hygrométrique a varié de 45 à $90 \%$ (moyennes journalières) et tendait à s'élever avec le temps au cours de la seconde série expérimentale.

\section{c / Mesures et analyses}

Les poulets ont été pesés par groupe toutes les deux semaines. La consommation alimentaire a été mesurée toutes les 4 semaines en groupes (sexes réunis) et l'efficacité alimentaire par périodes de 28 jours en a été déduite. La mortalité a été relevée quotidiennement.

En ce qui concerne les poids corporels, une analyse de variance a été faite selon le type génétique, le sexe et la teneur en vitamine $\mathrm{C}$ de l'aliment, mais ce dernier facteur n'ayant entraîné aucun effet significatif, nous ne présentons ici que les effets moyens du type génétique, pour les deux sexes réunis.

\section{2 / Résultats et discussion}

La différence entre types génétiques (tableau 1), sexes réunis, est hautement significative à tous les âges pour les poids, les consommations alimentaires et les indices de consommation, à l'avantage du croisement ISA715 (figure 1). En revanche le taux de mortalité a été significativement plus élevé pour ce croisement commercial.

\section{a / Niveau des performances}

Il faut tenir compte des conditions particulières d'environnement (température pour le 2è essai ; aliment modérément énergétique) pour apprécier le niveau des performances. Dans l'ensemble l'état sanitaire s'est montré satisfaisant et la mortalité faible, en dépit des températures ambiantes souvent élevées au cours de l'essai en saison chaude, et, en ce qui concerne le premier essai, en dépit d'un délai anormal dans le transport aérien des poussins.

\section{b / Effet de la saison}

Pour les deux types génétiques la croissance est nettement moins bonne en saison chaude (série née en juin), très vraisemblablement du fait des températures plus élevées en juinjuillet. Les indices de consommation, par contre, ont été relativement peu affectés. Dans les premières semaines, les indices élevés (donc médiocres) du premier essai pourraient être un effet du mode de chauffage qui a conduit à des températures initiales asssez faibles, ce qui n'a pas été le cas en saison chaude.

\section{c / Comparaison entre les deux croisements dans chaque essai}

Les deux croisements comparés dans les deux séries d'élevage réalisés étaient de type très différent, "Label" d'une part, poulet de chair standard de l'autre. Même relativement lourd pour un "Label", le premier croisement a dans les deux essais un poids moyen nettement plus faible que l'autre. Il ne s'agissait pas de mettre en compétition deux produits destinés à des objectifs différents, mais il est intéressant de constater que l'effet de la température est moindre pour le croisement 431 que ce soit pour le poids (figure 1) ou pour l'indice de consommation (tableau 1). Peutêtre l'écart de poids aurait-il diminué encore au-delà de 49 jours, mais, pour des raisons matérielles, le second essai n'a pu être mené au-delà. La réduction du handicap du croisement "Label" en saison chaude s'observe aussi sur les indices de consommation.

Figure 1. Variation relative des poids corporels de la saison froide à la saison chaude.

poids (

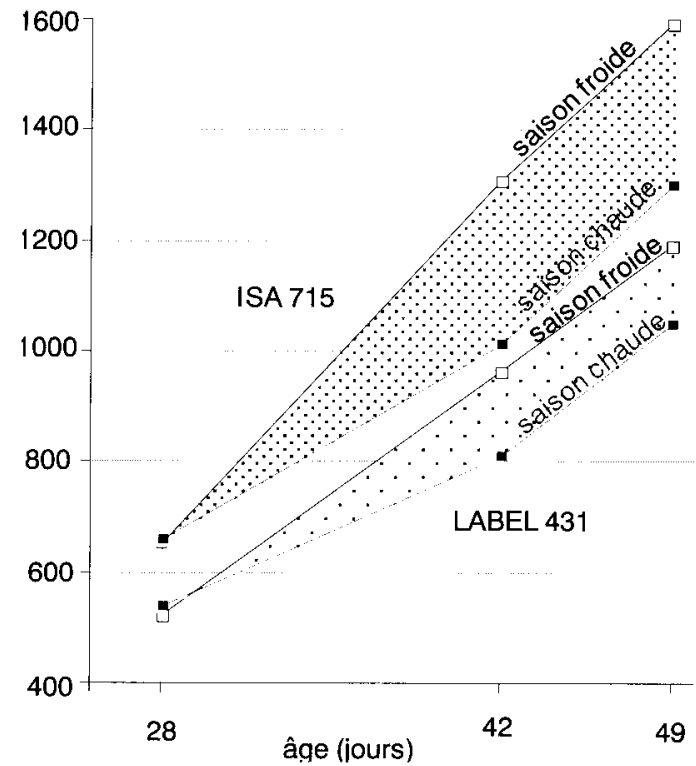


Tableau 1. Croissance et efficacité alimentaire comparées des deux croisements (chair) dans 2 essais successifs.

\begin{tabular}{|c|c|c|c|c|c|c|c|c|c|}
\hline & & \multicolumn{4}{|c|}{ Saison froide } & \multicolumn{4}{|c|}{ Saison chaude } \\
\hline & & ISA715 & SASSO 431 & différen & $\begin{array}{c}\text { ASSO-ISA } \\
\% \text { ISA }\end{array}$ & ISA715 & SASSO431 & différence & $\begin{array}{l}\text { ASSO-ISA } \\
\% \text { ISA }\end{array}$ \\
\hline \multicolumn{10}{|c|}{ Poids corporel moyen (g) (sexes réunis) } \\
\hline âge (j) & $\begin{array}{l}14 \\
28 \\
42 \\
49 * \\
56 \\
70\end{array}$ & $\begin{array}{c}223 \\
653 \\
1308 \\
(1593) \\
1878 \\
2387\end{array}$ & $\begin{array}{c}191 \\
520 \\
963 \\
(1190) \\
1416 \\
1886\end{array}$ & $\begin{array}{r}-31 \\
-133 \\
-344 \\
- \\
-462 \\
-501\end{array}$ & $\begin{array}{c}-14,1 \\
-20,4 \\
-26,4 \\
- \\
-24,6 \\
-21,0\end{array}$ & $\begin{array}{r}251 \\
654 \\
1008 \\
1297\end{array}$ & $\begin{array}{r}215 \\
534 \\
804 \\
1044\end{array}$ & $\begin{array}{r}-36 \\
-120 \\
-204 \\
-253\end{array}$ & $\begin{array}{l}-14,3 \\
-18,3 \\
-20,2 \\
-19,5\end{array}$ \\
\hline \multicolumn{10}{|c|}{ Indice de consommation (g aliment / g gain de poids) } \\
\hline \multicolumn{2}{|c|}{$\begin{array}{l}0-28 \mathrm{j} \\
0-49 \mathrm{j} \\
0-56 \mathrm{j} \\
0-70 \mathrm{j}\end{array}$} & $\begin{array}{c}3,31 \\
- \\
2,55 \\
2,79\end{array}$ & $\begin{array}{c}3,64 \\
- \\
2,84 \\
3,05\end{array}$ & \multicolumn{2}{|c|}{$\begin{array}{c}+0,33 \\
- \\
+0,29 \\
+0,26\end{array}$} & $\begin{array}{l}1,90 \\
2,49\end{array}$ & $\begin{array}{l}2,06 \\
2,69\end{array}$ & \multicolumn{2}{|c|}{$\begin{array}{l}+0,16 \\
+0,20\end{array}$} \\
\hline \multicolumn{10}{|c|}{ Mortalité (\%) } \\
\hline \multicolumn{2}{|c|}{$\begin{array}{l}0-49 \mathrm{j} \\
0-56 \mathrm{j} \\
0-70 \mathrm{j}\end{array}$} & $\begin{array}{c}- \\
7,9 \\
8,7\end{array}$ & $\begin{array}{l}- \\
1,1 \\
1,9\end{array}$ & & & $\begin{array}{c}2,2 \\
- \\
-\end{array}$ & $\begin{array}{c}1,3 \\
- \\
-\end{array}$ & & \\
\hline
\end{tabular}

$\left({ }^{*}\right)$ estimation à partir de la moyenne des poids à 42 et 56 jours pour le premier essai

On retrouve ceci d'une autre façon en comparant (tableau 1) la réduction de croissance obtenue dans le 2è essai comparé au premier, par âge, pour chaque croisement : aux âges de 42 et 49 jours, cette réduction est voisine de 23 et $19 \%$ respectivement pour le croisement 715 et de 16 et $12 \%$ respectivement pour le croisement 431 . Il paraît vraisemblable que le gène "Cou nu", présent dans le type "Label" rende compte de tout ou partie de cette différence (voir Mérat 1986). La taille inférieure du croisement 431 à âge égal peut jouer aussi un rôle (on sait que les oiseaux de plus petite taille tendent à mieux supporter les fortes chaleurs).

Enfin, dans tous les cas, la mortalité est un peu moindre pour les poulets 431 que pour le croisement 715 , y compris dans les premiers jours d'élevage du premier essai, suite au retard déjà signalé dans le transport aérien, retard que les poussin "Label" ont mieux supporté : 2 morts pour le croisement 431 et 40 pour le 715 . Toutefois, cet avantage n'est pas accentué en saison chaude, et comme dans l'ensemble les taux de mortalité n'étaient pas très élevés, ceci ne peut contrebalancer l'avantage général du type industriel quand à la croissance.

Cette comparaison utilisait des croisements disponibles correspondant à des types très différents. Les résultats montrent que, même dans les conditions locales réalisées avec leurs particularités, ils doivent être réservés à des élevages et à des marchés entièrement différents.

La comparaison faite à deux saisons pour les mêmes types génétiques nous a d'autre part permis d'observer que le croisement "Label" est relativement moins affecté par les conditions climatiques estivales que le croisement "standard" en ce qui concerne la croissance. Les données présentées jointes à des travaux antérieurs permettent d'attribuer ceci peut être partiellement au poids plus léger du type "label", mais aussi à son plumage moins isolant (type Cou nu). L'incorporation de la mutation correspondante dans une lignée parentale "poulet de chair classique" pourrait apporter à cette dernière un avantage relatif au moins en saison chaude.

\section{2 / Poules pondeuses}

\section{1 / Conditions expérimentales}

\section{a / Types génétiques comparés}

Le croisement testé était obtenu à partir de mâles d'une lignée expérimentale "naine Cou nu" (gènes $\mathrm{dw}$ lié au sexe et $\mathrm{Na}$ ) type "oeuf brun" (INRA, Laboratoire de Génétique Factorielle) sélectionnée pour des critères de production d'oeufs. Ces coqs étaient croisés à des poules ISA-Brown (croisement terminal).

Un lot du même croisement terminal ISA éclos le même jour que le premier croisement servait de témoin "commercial" (type pondeuse à oeufs bruns).

\section{$b$ /Conditions d'élevage}

L'éclosion a eu lieu le 18/01/1990 et les poussins ont été transférés par avion de Jouyen-Josas à Rabat.

Les poulettes étaient élevées dans une poussinière avec fenêtres sur deux niveaux, au sol sur litière de paille, la densité étant d'environ 
$9 / \mathrm{m}^{2}$. Chaque type génétique était réparti dans une loge séparée. Un aliment commercial "croissance" était donné ad libitum à tous les poussins jusqu'à l'âge de 8 semaines, puis un aliment "poulettes" de 8 à 20 semaines, avec pour les poulettes ISA, un rationnement "ship-a-day".

Les poussins étaient vaccinés contre la maladie de NewCastle, la bronchite infectieuse et la maladie de Gumboro, la variole, l'encéphalomyélite et plusieurs maladies parasitaires.

L'éclairement, de 24 heures par jour les trois premiers jours, passait ensuite à $14 \mathrm{~h} 30$, puis les poulettes étaient graduellement amenées à $16 \mathrm{~h} 30$ selon le programme lumineux adapté au croisement ISA. L'intensité lumineuse correspondait à une puissance de $4 \mathrm{w} / \mathrm{m}^{2}$.

Les poulettes étaient transférées en cages de ponte à l'âge de 20 semaines. Ces cages, d'un type commercial, contenaient chacune 3 poules du même type jusqu'à l'âge de 40 semaines. A cet âge, pour des raisons matérielles, les poules étaient mises à 4 par cage (au total 90 par type génétique).

L'aliment donné aux pondeuses, en farine, contenait $16,5 \%$ de matières azotées, 2850 kcal d'énergie métabolisable/kg et 3,5\% de calcium. Après le pic de ponte, les poules ISA étaient rationnées à $120 \mathrm{~g} / \mathrm{jour}$ environ (moyenne par poule), l'autre croisement continuant à recevoir l'aliment ad libitum.

L'éclosion, en janvier, était suivie de températures modérées en poussinière. Les températures s'élevaient dans la période précédant et suivant l'entrée en ponte, qui se situait en juin-juillet. Les températures moyennes journalières pour la période juin-juillet-août étaient comprises entre $25^{\circ}$ et $34^{\circ} \mathrm{C}$, puis elles décroissaient.

\section{c / Mesures et analyses}

Le pourcentage de ponte par périodes est basé sur les poules présentes durant chaque période. Le poids moyen des oeufs porte sur tous les oeufs de chaque poule durant 3 jours consécutifs dans la période correspondante. La masse d'oeufs est estimée comme le produit du pourcentage de ponte par le poids moyen des oeufs. L'indice de consommation est déduit de la consommation journalière et de la masse d'oeufs. Les caractéristiques de qualité des oeufs sont mesurées sur 3 oeufs consécutifs par poule pour chaque période. Les coquilles ont été lavées puis séchées à température ambiante et l'épaisseur a été déterminée avec les membranes coquillières à l'aide d'un micromètre dans la zone équatoriale de l'oeuf. La densité a été déterminée par flottation dans des solutions salines $(\mathrm{NaCl})$ de concentration croissant de 0,005 allant de 1,065 à 1,100 .

Pour chaque caractère et chaque période, les deux croisements étaient comparés par un test. Un apport différent de vitamine C à la ration de base n'ayant donné lieu à aucune différence significative, il n'est pas tenu compte de ce facteur dans la présente analyse.

\section{2 / Résultats et discussion}

Les performances de ponte et les consommations sont présentées au tableau 2 qui donne l'évolution dans le temps et la moyenne d'ensemble par type génétique. Le tableau 3 donne seulement les moyennes générales des caractéristiques de qualité de l'oeuf, car celles-ci n'ont pas varié de façon appréciable au cours de la période de mesure.

Outre ces paramètres de productivité, la mortalité depuis la mise en cages jusqu'à l'âge de 40 semaines a été enregistrée. Elle était de $11,0 \%$ et $18,9 \%$ pour les poules naines $\mathrm{Cou}$

Tableau 2. Comparaison des deux types génétiques pour leurs performances de ponte et d'efficacité alimentaire à divers âges.

\begin{tabular}{|c|c|c|c|c|c|c|c|c|c|c|}
\hline \multirow{2}{*}{$\begin{array}{c}\text { Age ou } \\
\text { intervalle } \\
\text { (semaines) }\end{array}$} & \multicolumn{2}{|c|}{$\begin{array}{l}\text { Pourcentage } \\
\text { de ponte }\end{array}$} & \multicolumn{2}{|c|}{$\begin{array}{l}\text { Poids moyen } \\
\text { des œufs (g) }\end{array}$} & \multicolumn{2}{|c|}{$\begin{array}{l}\text { Poids corporel } \\
\text { (g) }\end{array}$} & \multicolumn{2}{|c|}{$\begin{array}{c}\text { Consommation } \\
(\mathrm{g} / \mathrm{j})\end{array}$} & \multicolumn{2}{|c|}{$\begin{array}{c}\text { Efficacité } \\
\text { alimentaire }\end{array}$} \\
\hline & $\begin{array}{l}\text { naines } \\
\text { Cou nu }\end{array}$ & ISA & $\begin{array}{l}\text { naines } \\
\text { Cou nu }\end{array}$ & ISA & $\begin{array}{l}\text { naines } \\
\text { Cou nu } \\
\end{array}$ & ISA & $\begin{array}{l}\text { naines } \\
\text { Cou nu }\end{array}$ & ISA & $\begin{array}{l}\text { naines } \\
\text { Cou nu }\end{array}$ & ISA \\
\hline 10 & , & & & & 659 & $962 * * *$ & & & & \\
\hline 12 & & & & & 800 & $1032^{* * *}$ & & & & \\
\hline 19 & & & & & 1066 & $1553^{* * *}$ & & & & \\
\hline 22 à 25 & 67,6 & $51,8^{* * *}$ & & & & & & & & \\
\hline 26 à 30 & 81,3 & $78,7 \mathrm{~ns}$ & & & & & & & & \\
\hline 35 ou $31-35$ & 75,2 & $75,0 \mathrm{~ns}$ & 52,2 & $57,2 * * *$ & 1363 & $1677^{* * * *}$ & 99,5 & 110,4 ** & $(2,53)^{1}$ & $(2,57)$ \\
\hline $36-40$ & 78,0 & $81,5 \mathrm{~ns}$ & 56,1 & $61,6 * * *$ & & & 96,8 & $108,2^{* * *}$ & $(2,21)$ & $(2,16)$ \\
\hline $41-44$ & 72,9 & $78,5^{* *}$ & 55,4 & $62,8^{* * *}$ & 1477 & $1841^{* * *}$ & 101,4 & $114,1^{* * *}$ & $(2,51)$ & $(2,33)$ \\
\hline $45-48$ & 71,1 & $80,6 * * *$ & 57,5 & $63,2^{* * *}$ & & & 94,2 & $104,8^{*}$ & $(2,31)$ & $(2,20)$ \\
\hline $49-52$ & 68,8 & $83,3^{* * *}$ & 58,4 & $65,7^{* * *}$ & & & 97,3 & $120,3 * * *$ & $(2,48)$ & $(2,30)$ \\
\hline $53-55$ & 65,8 & $81,5^{* * *}$ & 59,7 & $68,5^{* * *}$ & 1530 & $1952^{* * *}$ & 99,3 & $126,5^{* * *}$ & $(2,69)$ & $(2,41)$ \\
\hline $56-60$ & 63,6 & $77,1^{* * *}$ & & & & & & & & \\
\hline $61-65$ & 60,0 & $65,4^{* * *}$ & 58,4 & $63,6^{* * *}$ & 1435 & $1782^{* * *}$ & & & & \\
\hline Total & 70,4 & $75,3^{* * *}$ & 56,8 & $63,1^{* * *}$ & & & 98,1 & $114,1^{* *}$ & & \\
\hline
\end{tabular}

à partir de la masse d'œufs estimée dans la période correspondante (consommation/masse d'œufs) 
nu et ISA (différence non significative). De 40 à 66 semaines, les valeurs correspondantes pour les mêmes types génétiques étaient respectivement 11,0 et $21,3 \%$ (différence non significative).

\section{a / Niveau des performances}

Ce niveau peut être considéré comme satisfaisant, tant du point de vue des taux de ponte obtenus que de la viabilité, et compte tenu des fluctuations climatiques de la période d'été.

\section{b / Comparaison des types génétiques}

Pour les performances de ponte, on pouvait attendre une supériorité globale du croisement commercial, sur un croisement dont l'un des parents ne prétendait pas au même niveau. D'autre part, l'interprétation suggérée tient compte de la présence dans le croisement expérimental des gènes dw et $\mathrm{Na}$ dont les effets propres ont été étudiés antérieurement.

Pour le début de ponte, le croisement expérimental se révèle quelque peu avantageux par rapport au type "commercial" pour le taux de ponte et l'indice de consommation. Il est inférieur pour le poids moyen de l'oeuf, en relation avec la taille corporelle. Cependant, un poids moyen de l'ordre de $55 \mathrm{~g}$ en début de ponte reste acceptable.

$\mathrm{La}$ présence du gène $\mathrm{Na}$ (cou nu) dans le premier croisement peut peut-être rendre compte de son intensité de ponte comparativement bonne. Ce croisement semble moins affecté par les "coups de chaleur" (dont 3 maxima supérieurs à $40^{\circ} \mathrm{C}$ entre les âges de 25 et 28 semaines). Dans ce même croisement le taux de ponte ne paraît pas affecté par le gène de réduction de taille $\mathrm{dw}$, en accord avec d'autres observations tendant à montrer que les effets dépressifs du gène $d w$ sur la ponte sont peu marqués en croisement.

Après l'âge d'environ 40 semaines, l'avantage du croisement commercial s'exprime par une chute plus lente de la courbe de ponte, ce qui entraîne des indices de consommation plus favorables (de 5 à $10 \%$ selon les périodes) sauf en fin de ponte où la différence d'intensité de ponte se réduit et où les indices de consommation redeviennent favorables au croisement de plus petite taille.

Pour la période de contrôle (de l'entrée en ponte à l'âge de 65 semaines), la valeur moyenne des poules "naines Cou nu" exprimée en $\%$ des ISA est $93,3 \%$ pour le pourcentage de ponte, $90,1 \%$ pour le poids des oeufs, $86,0 \%$ pour la consommation d'aliment. L'indice de consommation estimé montre un désavantage des "naines Cou nu" d'environ $2 \%$ dans l'ensemble.

Le tableau 3 montre une valeur moyenne significativement plus élevée du croisement ISA pour l'épaisseur de la coquille et pour la hauteur de l'albumen, différences explicables
Tableau 3. Valeurs moyennes des paramètres de qualité de l'œuf (mesures échelonnées entre 35 et 65 semaines).

\begin{tabular}{|l|c|c|c|}
\hline \multicolumn{1}{|c|}{ Valeur moyenne } & $\begin{array}{c}\text { Naines } \\
\text { Cou nu }\end{array}$ & ISA & Signif. \\
\hline Densité & 1,087 & 1,089 & $\mathrm{~ns}$ \\
Hauteur de l'albumen $(\mathrm{mm})$ & 6,44 & 7,27 & $* * *$ \\
Unités Haugh & 80,0 & 81,9 & $\mathrm{~ns}$ \\
Epaisseur coquille $(0,01 \mathrm{~mm})$ & 39,9 & 41,4 & $* *$ \\
\hline
\end{tabular}

par le poids de l'oeuf plus élevé dans ce croisement. De fait les deux autres variables, densité et unités Haugh, indépendantes de ce poids, ne diffèrent pas entre types génétiques.

Ce bilan est peu défavorable au type génétique "expérimental" pour l'efficacité alimentaire. Il l'est davantage pour la production d'oeufs en fin de ponte et pour le poids moyen des oeufs. Il faudrait cependant tenir compte du rationnement appliqué au type "commercial" qui réduit les différences de consommation d'aliment entre croisements et sans lequel l'avantage des ISA pour l'indice de consommation serait sans doute annulé. On pourrait ajouter que le programme lumineux était adapté spécifiquement au croisement ISA. D'autre part, le bilan qui précède ne prend pas en compte la mortalité, apparemment moins élevée pour les poules naines Cou nu, ni le pourcentage d'oeufs cassés, qui n'a pu être enregistré, mais on sait que la présence du gène $d w$ abaisse en général ce pourcentage (Mérat 1990). L'avantage connu des gènes $\mathrm{Na}$ et peut être dw pour la thermotolérance peut expliquer une meilleure survie des poules portant ces gènes.

\section{Remerciements}

Ce travail fait en coopération entre le Laboratoire de Génétique Factorielle - INRA., Jouy-en-Josas (France) et l'Institut Agronomique et Vétérinaire Hassan II, Rabat (Maroc), a fait l'objet d'un contrat accordé par la Commission des Communautés Economiques Européennes (CEE.), au titre des actions "Sciences et Techniques au service du Développement".

Nous remercions d'autre part la SASSO (Sélection Avicole de la Sarthe et du Sud-Ouest, 40630 Sabres) de sa coopération pour la mise en place du croisement et l'envoi de poussins "Label".

\section{Références bibliographiques}

Mérat P., 1986. Potential usefulness of the Na (Naked neck) gene in Poultry production World's Poult. Sci. J. 42, 124-142.

Mérat P., 1990. Pleiotropic and associated effects of major genes. In Crawford R.D., ed. Poultry Breeding and genetics, p. 429-467. Elsevier, Amsterdam.

Rauen H.W., Horst P., Valle-Zarate A., 1986. Bedeutung des gens für Befiederungsreduktion und Nackthalsigkeit (Na) für die produktive Adaptationsvermögen von Legehennen unter hoher Temperatur Dauerbelastung. Archiv f. Geflügelk, 50, 235-245. 


\section{Summary}

Test of genetic types for a local poultry production in Morocco

A cross of a "Label" naked-neck type and a standard commercial broiler cross were both tested in typical husbandry and climatic conditions in Morocco, first in winter (hatching date 10.01.90), then in the warm season (hatching date 05.06.90). Body weight, feed efficiency and mortality were recorded up to the age $70 \mathrm{~d}$ in the former case, $49 \mathrm{~d}$ in the latter. The growth performances of the two crosses, as expected, were greatly different. The negative effect of the warm season on body weight and feed efficiency was less marked for the Label" type, which in addition showed very low mortality in all cases.

On the other hand an experimental cross of the brown-egg layer type including the sex-linked dwarf (dw) and Naked neck ( $\mathrm{Na}$ ) genes was tested for egg production in typical conditions of Morocco, together with a commercial (isa-brown) brown-egg layer cross. As expected, the laying persistency and mean egg weight of the experimental cross were inferior to those of the commercial cross. Conversely, the lower body weight and feed consumption of the former cross contributed to reducing the disadvantage of this cross for feed conversion, together with a slightly higher laying rate up to the age of 35 weeks, probably associated with a favorable effect of the dw and Na genes on heat tolerance. The data suggest a lower mortality rate for the dwarf naked neck cross after the age 40 weeks.

BENABDELJELIL K., MERAT P. 1992. Test de types génétiques pour une production avicole locale au Maroc. INRA Prod. Anim., 5 (3), $173-178$. 